Vinculan la ansiedad intensa con el riesgo de morir en adultos mayores de una población multiétnica

Entre 10 y $20 \%$ de los adultos mayores presentan síntomas de ansiedad. Sin embargo, aún no se han estudiado a cabalidad todas las aristas de este trastorno.

Se realizó un estudio longitudinal de 5 años basado en la población para determinar si hay asociación entre la ansiedad y el riesgo instantáneo de morir en adultos mayores. Para ello se seleccionaron 506 personas de 75 años o más que vivían en la comunidad en el condado de Galveston, Texas, Estados Unidos de América (176 personas caucásicas de origen no hispano, 177 afroamericanos de origen no hispano y 153 hispanos). Mediante técnicas de muestreo diferencial se seleccionaron conjuntos similares de cada grupo étnico con igual número de hombres y mujeres. La selección se hizo a partir de la base central de datos de beneficiarios de Medicare en el condado. Se utilizó la escala de Zung para la autoevaluación de la ansiedad. Las muertes se contabilizaron a partir de los registros del Índice Nacional de Muertes de los años 1995-1999.

En el período estudiado murieron 179 (36\%) de las personas estudiadas, de ellas 53 caucásicos no hispanos, 70 afroamericanos no hispanos y 56 hispanos. Las causas de muerte más frecuentes fueron: trastornos cardiovasculares $(n=85)$, cáncer $(n=36)$ e influenza o neumonía $(n=15)$. Entre las restantes causas estaban la diabetes, la demencia, los trastornos renales y los accidentes.

Se encontró que la ansiedad estaba asociada con el grupo étnico $(P=0,0001)$. Una mayor proporción de caucásicos no hispanos de mayor edad presentaron ansiedad intensa (puntuación > 50) en comparación con los afroamericanos no hispanos y los hispanos, que presentaron resultados similares. También se observó una asociación significativa entre la ansiedad intensa y el informe de dos o más trastornos de salud $(31,8 \%)$, la autoevaluación de mala salud $(35,3 \%)$ y tener limitaciones para realizar al menos una actividad de la vida diaria $(30,2 \%)$.

La ansiedad intensa estuvo asociada con un mayor riesgo instantáneo de morir por cualquier causa. Al ajustar según la edad y el sexo, el cociente de riesgos instantáneos (hazard ratio, HR) en personas con ansiedad intensa fue de 1,66 (intervalo de confianza de 95\% [IC95\%]: 1,14 a 2,42). Ajustes adi- cionales según el grupo étnico, el nivel de educación, el estado civil, la condición de fumador, el consumo de alcohol y el número de trastornos médicos informados no afectaron a esa asociación ( $\mathrm{HR}=1,56$; IC95\%: 1,05 a 2,32). Tampoco la autoevaluación de mala salud y el número de limitaciones para realizar actividades de la vida diaria cambiaron la significación de la asociación entre la ansiedad intensa y el riego instantáneo de morir ( $\mathrm{HR}=1,52$; IC95\%: 1,02 a $2,28)$. Por cada punto que aumentó la evaluación de la ansiedad, se incrementó en $2 \%$ el riesgo instantáneo de morir ( $\mathrm{HR}=1,02$; IC95\%: 1,00 a 1.05).

La ansiedad intensa también se asoció significativamente con el riesgo instantáneo de morir por trastornos cardiovasculares (HR = 1,90; IC95\%: 1,06 a 3,38) o por cáncer (HR = 2,38; IC95\%: 0,88 a 6,45). Por cada punto que aumentó la evaluación de la ansiedad se incrementó el riesgo de morir por trastornos cardiovasculares (HR $=1,03$; IC95\%: 1,00 a 1,07) o por cáncer (HR = 1,03; IC95\%: 0,99 a 1,09).

Este estudio demostró que la ansiedad intensa en personas de 75 años o más estaba asociada con un mayor riesgo instantáneo de morir por cualquier causa y, específicamente, por trastornos cardiovasculares o cáncer en los 5 años de seguimiento, independientemente de los factores de riesgo. Debido a la alta prevalencia de ansiedad en los adultos mayores y tomando en cuenta que existen intervenciones eficaces para controlar esta afección, el perfeccionamiento y la aplicación de las técnicas de tamizaje pueden tener un impacto considerable para mejorar la salud y prolongar la vida de las personas de mayor edad. (Ostir GV. High anxiety is associated with an increased risk of death in an older tri-ethnic population. J Clin Epidemiol. 2006;59(5):534-40.)

\section{El abandono súbito del hábito de fumar afecta a la memoria activa de los fumadores}

Estudios realizados indican que el tabaquismo crónico, el abandono súbito de ese hábito, la administración de nicotina y el fumar intensamente pueden influir negativamente en los resultados de las pruebas de funcionamiento de la memoria activa. Sin embargo, las opiniones al respecto son contradictorias.

Este artículo examina el efecto de fumar cigarrillos y de abandonar súbitamente ese hábito sobre 